\title{
An Acknowledgement to the HCA Healthcare Journal of Medicine's Initial Cohort of Reviewers, Authors, Editors and Advisory Board
}

Bruce Deighton, PhD, ${ }^{1}$ Hale Z. Toklu, PhD, MPharm, ${ }^{1,2}$ Graig Donini ${ }^{1}$

\section{Abstract}

\section{Description}

As the HCA Healthcare Journal of Medicine reaches the half way point of our first year in publication, we want to thank those who have provided invaluable support for the journal since the first submissions arrived in November of last year. The successful launch of our journal would not have been possible without the assistance of our reviewers, authors and board members.

\section{Keywords}

scholarly communication; peer review, research; open access publishing; education, medical, graduate; medical journalism

"Publish houses of brick, not mansions of straw." Dr. William G. Kaelin Jr., 2019's Nobel Laureate for Physiology or Medicine and Professor of Medicine at Harvard University and the Dana-Farber Cancer Institute, wrote this directive to researchers in his 2017 article in Nature. We noted in the journal's opening editorial, we want to make the HCA Healthcare Journal of Medicine a "house of bricks". ${ }^{2}$ The authors, reviewers and editors will provide the "bricks", through their submissions and the rigorous process of peer review and the careful eye of our editors.

As we reach the half way point of our first year in publication, we want to thank those who have provided invaluable support for the journal since the first submissions arrived in November of last year. The successful launch of our journal would not have been possible without the assistance of our reviewers, authors and board members. In this group are many who have authored and reviewed articles or served in the scientific boards of the journals in the past. For whom this experience was new, we hope that you found this experience an interesting one and decide to continue to work with us as we develop the journal into a worldclass publication.

For our colleagues in Graduate Medical Education, authorship and peer review are forms of scholarly activity recognized by the ACGME. Teaching residents on the finer points of your craft on the ground is important, but honing your critical thinking skills by engaging in scholarship is of increasing importance for a comprehensive training regimen for your residents. This journal isn't just about sharing the pursuits of HCA Healthcare Graduate Medical Education, we also share the quality work of faculty, physicians, and others doing medical research and clinical practice globally. Our authors and reviewers come from many diverse backgrounds from around the world and we hope in the years ahead this publication will continue to grow to be a service to the worldwide community of clinical practitioners.

Thank you for your support of the HCA Healthcare Journal of Medicine!

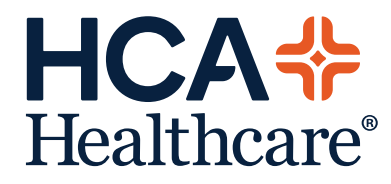

www.hcahealthcarejournal.com

(c) 2020 HCA Physician Services, Inc. d/b/a Emerald Medical Education
HCA Healthcare Journal of Medicine 
Reviewers

Khalid Abusaada

Eric Aguila

Ahmet Akici

Thomas Alexander

Clara L. Alvarez Villalba

Napatkamon Ayutyanont

J. David Baxter

Scarlet Benson

Andrea Berry

Steve Borzak

Christopher L. Bray

Isabel Jeannette Brea

David B. Byck

Steven Lee Carpenter

Kevin Carr

Mike Churuba

Jake Cho

Cristobal Cintron

Robyn Cobb

Christopher Corbit

William Cox

Ettore Crimi

Diane Davey

William B. DeHart

Nilufer N. Turan Dural

Oliwier Dziadkowiec

Anthony Espinoza

Michael Falgiani

\section{Authors}

Hossein Akhondi

Omar Ansari

Ranjit S. Banwait

Jo Cooke-Barber

Marissa Bournique

Jared Brackenrich

Cristobal Cintron

Robert Dean Chait

Alex Chao

Yasna Chaudhary

Brendon T. Cornett

Allison Dalton

William Brady DeHart

Bruce Deighton

Nakeya Dewaswala

Graig Donini

Jeffery S. Durbin

Oliwier Dziadkowiec

Julia Fashner

Troy J. Fishman

Alvaro Frometa

Barbara L. Gracious

Scott Gutovitz
Julia Fashner

Mike Flynn

Jacklyn C. Fuller

Ankur Garg

Lucio Gordan

Barbara L. Gracious

Shaikh A. Hai

Tayfun Hakan

Jacqueline Halladay

Karen E. Harris

Robyn Hoelle

Afra Janarious

Jennifer Jones

Mohamad Kabach

Olga Karasik

Daniel Kaswan

Mustafa Kinaan

John S. Kirkland

Philip Kondylis

Kenneth Lan

Scott Lang

Se Won Lee

Tracy Macintosh

Machaiah Madhrira

Sharmineh Mansoori

Anuja S. Mehta

Dean McCarley

Melissa Mendez

Diana Mora-Montero

Julie A. Jacko

Mike Hansen

Sarah E. Hatef

Ashan T. Hatharasinghe

Robyn Hoelle

Nannette Hoffman

Uma G. lyer

Nigel Jagoo

Dietrich Jehle

Chad Johnston

lqbal Kapadia

Nicole Kassen

Kimball C. Kaufman

Shobhit Keswani

Breonna Kinnison

Emmanouil Kiriakopoulos

Seth Kozlowski

Phuong U. Le

Samuel M. Leaman

Se Won Lee

Thomas Martel

Rabia Mazhar

Analise McGreal

Monica N. Melmer
Jonathan Pangia

Rajul Parikh

Anthony Pearson-Shaver

Anush S. Pillai

Kanakadurga Rao Poduri

Rakesh Prashad

David M. Quillen

Dan Roberts

Dipali R. Sahoo

Ozer Sehirli

Pinak A. Shah

Anthony Shadiack

Eric K. Shaw

Joshua Shultz

Taeyong Sim

Stephen Spann

Bruce St. Amour

Shala Sundaram

Stephen Thacker

Timothy Tiu

Hale Z. Toklu

Robert L. Turner

Craig Van Dien

Salvador Villanueva

Steven Warrington

Daniel Witter

Sahzene Yavuz

Berrak C. Yegen

Jocelyn N. Mineo

Vignesh Jayaraman Muralidharan

Megan L. Novak

Christopher N. Ochner

Jonathan Pangia

Don Pepito

Vanessa Perez

Francois Sainfort

Joshua K. Salabei

Frederick M. Schiavone

Andrew Schwemmer

Christopher Senkowski

Bruce St. Amour

Justin Steinberg

Samantha Sternad

Timothy Tiu

Hale Z. Toklu

Craig Van Dien

Christian Vandever

Johannes Vieweg

Emilio G. Volz

Paula S. Wales

Tony Zitek 


\author{
Advisory Board \\ Khalid Abusaada \\ Michael Allswede \\ Anya Andrews \\ David Baxter \\ Steven Carpenter \\ Christopher Corbit \\ Nemer Dabage-Forzoli \\ Lora Denton \\ Donald Eagerton \\ Latha Ganti \\ Maryam Gondal \\ Joshua Hagan \\ Nannette Hoffman \\ Chris Kaufmann \\ Philip Kondylis \\ Merrill Krolick \\ Michaiah Madhrira \\ Melissa Mendez \\ Gerald O'Malley
}

Jonathan Pangia

Anush Pillai

David Quillen

Alfredo Rivera

Dipali Ruby Sahoo

Upinder Singh

Salim Surani

Mark Trolice

Stephen Weis

Albert Yurvati

Thomas Alexander

Clara Alvarez-Villalba

Scarlet Benson

Brian Childs

Ettore Crimi

Nicholas D'Angelo

Scott Duncan

Mike Flynn

Tarun Ghosh

Scott Gutovitz
Karen Harris

Jeffery Huang

Ethan Kass

John Kirkland

Karthik Krishnamurthy

Tulisa LaRocca

Shannon McNabb

Salman Muddassir

Michelle Ozcan

Michael Pesce

Rakesh Prashad

Jamal Rahimi

Javier Rosario

Eric Shaw

Shala Sundaram

Stephen Thacker

Steve Warrington

Brian Wood
Kamal Mohiuddin

Hiren Patel

Fergie Ramos Taurez

Mehboob Rehan

Youssef Shaban

Kara Stout

Pratikkumar Vekaria

Sahityan Viswanathan

Risiq Zackria

Niazi Anum

Kevin Carr

Nick Christenson

Sarah Graff

Bassam Hanna
Raul Isern

Andre Johnson

Mustafa Kinaan

John McNeil

Odinaka Mogor

Kristina Nakonechnaya

Brett Prestia

Abby Regan

Chandra Sanwal

Ashish Sharma

Kunjan Udani

Seetha Venkateswaran

Amanda Webb

\section{Editorial Board}

Alex Ahkondi

David Byck

Robert Chait

Julia Fashner

Barbara Gracious

Jacqueline Halladay

Julie Jacko

Sewon Lee

Anthony Pearson-Shaver

Emilio Volz

Marissa Bournique

Kenneth Lan
Clinical Editor

Hale Z. Toklu

Editor-In-Chief

Bruce Deighton

\author{
Production Editors \\ Jan Haley \\ Lindsay Million
}

\section{Managing Editor}

Graig Donini 


\section{Conflicts of Interest}

The authors declare they have no conflicts of interest.

Drs. Deighton and Toklu and Mr. Donini are employees of HCA Healthcare Graduate Medical Education, an organization affiliated with the journal's publisher.

This research was supported (in whole or in part) by HCA Healthcare and/or an HCA Healthcare affiliated entity. The views expressed in this publication represent those of the author(s) and do not necessarily represent the official views of HCA Healthcare or any of its affiliated entities.

\section{Author Affiliations}

1. HCA Healthcare Graduate Medical Education

2. University of Central Florida College of Medicine, Department of Clinical Science

\section{References}

1. Kaelin Jr WG. Publish houses of brick, not mansions of straw. Nature. 2017;(545):387387. https://doi.org/10.1038/545387a

2. Toklu HZ, Deighton B, Donini G. A New Journal to Publish Houses of Brick: Introducing the HCA Healthcare Journal of Medicine, HCA Healthcare Journal of Medicine. 2020;1(1):1-2. https://doi.org/10.36518/2689$\underline{0216.1046}$ 